\title{
Dificuldades das puérperas no aleitamento materno do seu primeiro filho em uma maternidade filantrópica do interior de Sergipe
}

\author{
Difficulties of puerperal women in breastfeeding their first child in a philanthropic maternity \\ hospital in Sergipe \\ Dificultades de las mujeres puerperales para amamantar a su primer hijo en una maternidad \\ filantrópica de Sergipe
}

Recebido: 29/06/2021 | Revisado: 06/07/2021 | Aceito: 09/07/2021 | Publicado: 21/07/2021

Ana Carla Silva Carvalho ORCID: https://orcid.org/0000-0001-7494-9532 Centro Universitário Estácio de Sergipe, Brasil E-mail: anacarlabbg@hotmail.com

Makson Mota de Jesus ORCID: https://orcid.org/0000-0002-7705-3931 Centro Universitário Estácio de Sergipe, Brasil E-mail: maksonmotta@ hotmail.com

Ruth Cristini Torres

ORCID: https://orcid.org/0000-0002-8664-192X Instituto de Hematologia e Hemoterapia de Sergipe, Brasil E-mail: ruthcristini@gmail.com

Weber de Santana Teles ORCID: https://orcid.org/0000-0003-1770-8278

Centro de Hemoterapia de Sergipe, Brasil E-mail: arteecura@hotmail.com

Max Cruz da Silva

ORCID: https://orcid.org/0000-0002-6944-5986

Faculdade Pio Décimo, Brasil

E-mail: maxlfi@hotmail.com

Ângela Maria Melo Sá Barros ORCID: https://orcid.org/0000-0003-4087-3247 Universidade Federal do Rio de Janeiro, Brasil E-mail: angelsamelo@ hotmail.com

Paulo Celso Curvelo Santos Junior ORCID: https://orcid.org/0000-0001-5834-6782 Universidade Tiradentes, Brasil E-mail: Paulo.curvelo.jr@gmail.com

Marcel Vinícius Cunha Azevedo ORCID: https://orcid.org/0000-0002-5312-3333 Centro Universitário Estácio de Sergipe, Brasil E-mail: marcelvinicius49@gmail.com

Ana Fátima Souza Melo de Andrade ORCID: https://orcid.org/0000-0002-7024-6175 Centro Universitário Estácio de Sergipe, Brasil E-mail: anafatimamelo@hotmail.com Alejandra Debbo ORCID: https://orcid.org/0000-0002-7743-5921 Universidade Tiradentes, Brasil E-mail: aledebbo@hotmail.com

Rute Nascimento da Silva ORCID: https://orcid.org/0000-0002-2719-1623 Universidade Tiradentes, Brasil E-mail: silva_rute@hotmail.com

Taíssa Alice Soledade Calasans ORCID: https://orcid.org/0000-0003-0460-4437 Universidade Tiradentes, Brasil E-mail: taissa.asc@gmail.com 


\begin{abstract}
Resumo
Objetivo: Identificar as dificuldades de mães internadas em uma maternidade do interior de Sergipe no aleitamento do seu primeiro filho. Metódos: Para análise quantitativa foram realizadas distribuições de frequências, por meio do programa Statistical Package For the Social Sciences for Windows- SPSS versão 21. Resultado: Constatou-se que todas alegam terem sido orientadas quanto aos benefícios do aleitamento materno e sobre os 10 passos para promover a amamentação, bem como 31 (50\%) afirmaram achar bom o ato de amamentar e $31(50 \%)$ relataram achar ótimo amamentar. Vale ressaltar que todos os recém-natos das puérperas do estudo permaneceram no alojamento conjunto durante todo o tempo de internação. Conclusão: Os resultados apontam para um resultado satisfatório em relação ao Aleitamento Materno Exclusivo e suas dificuldades no período estudado e evidenciou-se que o cenário do estudo realiza a orientação prévia das mães em relação ao processo de amamentação. A orientação adequada das mães sobre o AM é importante para o sucesso da amamentação no período pós-parto, amenizando possíveis problemas como dor na amamentação e desmame precoce.
\end{abstract}

Palavras-chave: Aleitamento materno; Leite humano; Orientação; Período pós-parto.

\begin{abstract}
Objective: To identify the difficulties of mothers hospitalized in a maternity hospital in the interior of Sergipe in breastfeeding their first child. Methods: For quantitative analysis, frequency distributions were performed using the Statistical Package for the Social Sciences for Windows- SPSS version 21 program. Result: It was found that all claimed to have been guided about the benefits of breastfeeding and about the 10 steps to promote breastfeeding, as well as 31 $(50 \%)$ stated that they thought breastfeeding was good and $31(50 \%)$ reported that breastfeeding was excellent. It is noteworthy that all newborns of the mothers in the study remained in rooming-in during the entire hospital stay. Conclusion: The results point to a satisfactory result in relation to Exclusive Breastfeeding and its difficulties during the study period, and it became evident that the study setting provides prior guidance to mothers in relation to the breastfeeding process. Adequate guidance of mothers about breastfeeding is important for successful breastfeeding in the postpartum period, alleviating possible problems such as pain during breastfeeding and early weaning.
\end{abstract}

Keywords: Breast feeding; Milk human; Orientation; Postpartum period.

\title{
Resumen
}

Objetivo: Identificar las dificultades de las madres hospitalizadas en una maternidad del interior de Sergipe para amamantar a su primer hijo. Métodos: Para el análisis cuantitativo, se realizaron distribuciones de frecuencia utilizando el Paquete Estadístico de Ciencias Sociales para Windows- SPSS versión 21 del programa. Resultado: Se encontró que todos afirmaron haber sido orientados sobre los beneficios de la lactancia materna y sobre los 10 pasos para promover lactancia materna, así como 31 (50\%) dijeron que pensaban que la lactancia materna era buena y 31 (50\%) informaron que la lactancia materna era excelente. Cabe señalar que todos los recién nacidos de las madres del estudio permanecieron en régimen de alojamiento conjunto durante toda la estancia hospitalaria. Conclusión: Los resultados apuntan a un resultado satisfactorio en relación a la Lactancia Materna Exclusiva y sus dificultades durante el período de estudio, y se evidenció que el escenario del estudio brinda orientación previa a las madres en relación al proceso de lactancia materna. La orientación adecuada a las madres sobre la lactancia materna es importante para una lactancia exitosa en el período posparto, aliviando posibles problemas como el dolor durante la lactancia y el destete temprano. Palabras clave: Lactancia materna; Leche humana; Guia; Período posparto.

\section{Introdução}

O protocolo de diretrizes de atendimento em aleitamento materno (AM) do departamento de atenção à saúde das pessoas do programa de aleitamento materno de Ribeirão Preto (2020), afirma que o aleitamento materno é a mais sabedora tática natural de vínculo, afetividade anteparo, alimentação e para o bebê estabelece a mais emotiva, barata e satisfatória para a minimização da morbimortalidade infantil.

O contato entre mãe e o neonato beneficia a aproximação, entretanto o apoio do profissional de saúde que atua diretamente neste serviço é de suma importância, um local admirável, acolhimento são fatores que ajudam a adaptação do neonato ao meio externo, estreitando os laços maternos propiciando benefícios na lactação (Rodrigues et al. 2020).

O leite produzido pelas puérperas sofre modificações em sua composição, tornando-se um alimento completo, com todos os nutrientes que o bebê necessita e a promoção da amamentação melhora a saúde e a qualidade de vida de toda a família. (Brasil, Ministério da Saúde. 2012) 
A orientação adequada para puérperas e gestantes sobre a amamentação colabora na sua segurança e motivação, pois falhar na amamentação mesmo com um forte desejo de efetivá-la, pode ser devido à falta de acesso a orientação ou apoio adequado de profissionais ou de pessoas mais experientes dentro ou fora da família.

Para que a amamentação seja uma prática frequente, é necessária a participação dos profissionais de saúde, apoiando e instruindo a nutriz durante o pré-natal e o alojamento conjunto, informando-a sobre a importância e vantagens dessa prática.

A enfermagem, nesse período, tem papel muito importante no ajuste da maternidade, com a educação em saúde, para que a mulher consiga alcançar, gradualmente, a autonomia do autocuidado e com o bebê, além de melhorar gradativamente a seu lado emocional, adequando a puérpera a fase da vida (Almeida et al. 2010).

Essas intensas modificações interferem não somente no fator físico da mulher, mas também no seu relacionamento interpessoal e familiar (Moura, Costa \& Teixeira, 2010). Nessa perspectiva, são essenciais os cuidados de enfermagem voltados à prevenção de complicações puerperais, oferecendo conforto físico e emocional e educação em saúde (Starpasson, 2010)

A Organização Mundial da Saúde (OMS) e o Ministério da Saúde (MS) recomendam que as crianças com até seis meses de vida devem ser alimentadas exclusivamente com leite materno. Orienta também a introdução de alimentos complementares após os seis meses de idade (Almeida et al. 2010).

Um programa Nacional idealizado pelo Ministério da Saúde, cujo o intuito é o incentivo do aleitamento materno, assim como mobilização dos profissionais da área de saúde e as coletividades, enfatizou que todos os envolvidos são responsáveis na promoção e ânimo ao aleitamento materno (Brasil. Ministério da Saúde, 2009).

Para a vitória do aleitamento materno os técnicos que operam no setor de saúde nas funções da redes dos municípios tem importância fundamental a coorporificar, agindo sobre subsequente com a ânimo de lactar, organização do aleitar e arrimo da amamentação (Del Castanhel, Delziovo \& Araújo, 2016).

Tendo em vista a importância do aleitamento materno para a criança e a mulher, entende-se que o sucesso do aleitamento materno está relacionado a programas educativos de incentivos ao Aleitamento Materno Exclusivo (AME), e aos hábitos culturais ligados a prática (Narchi et al. 2009).

Várias iniciativas são recomendadas sobre a alimentação infantil entre elas uma das mais importantes a Organização Mundial de Saúde (OMS), à Assembleia Mundial de Saúde, e os "Dez Passos para o Sucesso do aleitamento Materno", este último deu origem à estratégia Iniciativa Hospital Amigo da Criança (IHAC) (Miot, 2011). Seguindo as recomendações internacionais para uma pratica de sucesso no aleitamento materno e por seguir essas iniciativas em 1981 o Brasil iniciou o programa nacional de Incentivo ao aleitamento Materno (PNIAM) (Almeida et al. 2010).

O alactamento tem vultuosa função na diminuição das diferenças, auxiliando com a obtenção das metas de Desenvolvimento Sustentável, apresentados pela Organização das Nações Unidas, para que sejam atingidas até o ano de 2030 (Victoria et al., 2016).

O presente estudo apresenta relevância visto que é de suma importância que as mães entendam as vantagens da Amamentação Materna Exclusiva. Neste contexto, objetivou-se identificar as dificuldades de mães internadas em uma maternidade do interior de Sergipe no aleitamento do seu primeiro filho.

\section{Métodos}

Trata-se de um estudo descritivo, exploratório com abordagem quantitativa, com coleta de dados no período de agosto a setembro de 2018 realizado com puérperas internadas em uma maternidade do município de Itabaiana/Sergipe. O município está situado na região Agreste do estado, limita-se ao Norte com os municípios de Moita Bonita e Ribeirópolis, Campo do Brito e Itaporanga' Ajuda ao Sul, Ao Leste com os municípios de Areia Branca e Malhador e ao Oeste os municípios de Campo do brito, Macambira e Frei Paulo. Com cerca de 100 mil habitantes, Itabaiana é uma das maiores cidades do interior sergipano. 
Foram incluídas na amostra puérperas, maiores de 18 anos, mães do primeiro filho, internadas na referida maternidade. Como critério de exclusão está à recusa em participar da pesquisa e incapacidade de compreender os questionamentos. Os riscos da pesquisa serão de ordem emocional para as participantes, visto que as mesmas podem estar em um momento difícil diante da amamentação do primeiro filho.

A população desse estudo foi constituída por puérperas (um dia após o parto). Utilizou-se a técnica de amostragem por conveniência, por meio de convite para participação no estudo no período de agosto a setembro do ano de 2018.

Os dados foram coletados por meio de entrevista semiestruturada. Foi realizado um pré-teste do instrumento com 5 (cinco) puérperas da amostra visando identificar possíveis falhas e a realização das devidas correções antes da coleta de dados em toda a amostra. Para análise quantitativa foram realizadas distribuições de frequências, gráficos e tabelas por meio do programa Statistical Package For the Social Sciences for Windows- SPSS versão 21.

O estudo foi realizado de acordo de acordo com os princípios éticos da resolução 466/2012 do Conselho Nacional de Saúde para pesquisa com seres humanos, aprovado sob o parecer do Comitê de Ética em Pesquisa nº 848.627.

\section{Resultados}

Foram entrevistadas 62 puérperas com média de idade de 26,5 em média, com desvio padrão de 6,032. Em relação ao estado civil, $43(69,4 \%)$ eram casadas, $16(25,8 \%)$ solteiras e somente $3(4,8 \%)$ alegaram ser separadas. Cerca de $32(51,6 \%)$ tinham escolaridade de ensino fundamental incompleto ou completo, destas, $18(29 \%)$ eram lavradoras, $10(16,1 \%)$ cuidavam da casa, 2 (3,22\%) estudantes e 2 (3,22\%) possuíam outra ocupação.

Ao analisar possíveis problemas relacionados à amamentação vivenciados pelas puérperas, constatou-se que 46 (74\%) das pesquisadas não apresentaram problemas demonstrando um cenário favorável para a amamentação (Tabela 1).

Tabela 1 - Distribuição de puérperas em relação a amamentação anterior ou atual e problemas na amamentação. Aracaju, SE, 2018.

\begin{tabular}{cccccccc}
\hline \multirow{2}{*}{$\begin{array}{c}\text { Amamenta ou } \\
\text { amamentou } \\
\text { anteriormente? }\end{array}$} & $\begin{array}{c}\text { Abcesso } \\
\text { mamário }\end{array}$ & $\begin{array}{c}\text { Bloqueio de } \\
\text { ductos }\end{array}$ & Fissura & $\begin{array}{c}\text { Ingurgitamento } \\
\text { mamário }\end{array}$ & Nenhum & $\begin{array}{c}\text { Outra } \\
\text { situação }\end{array}$ & \\
\hline Sim & 1 & 4 & 5 & 3 & 46 & 1 & 60 \\
Não & 0 & 0 & 1 & 0 & 1 & 0 & 2 \\
\hline Total & 1 & 4 & 6 & 3 & 47 & 1 & 62 \\
\hline
\end{tabular}

Fonte: Autores.

Investigou-se a ocorrência de dor durante o ato de amamentar que foi relatada por apenas 11 puérperas $(17,7 \%)($ Tabela 2).

Tabela 2 - Distribuição de puérperas de acordo com a ocorrência e início da dor durante a amamentação. Aracaju, SE, 2018.

\begin{tabular}{cccccc}
\hline \multirow{2}{*}{ Dor na amamentação? } & \multicolumn{4}{c}{ Início da dor } & Total \\
\cline { 2 - 5 } & $\begin{array}{c}\text { Início da } \\
\text { mamada }\end{array}$ & $\begin{array}{c}\text { Durante a } \\
\text { mamada }\end{array}$ & $\begin{array}{c}\text { No intervalo da } \\
\text { mamada }\end{array}$ & N.A.* & \\
\hline Sim & 4 & 6 & 1 & 0 & 11 \\
Não & 0 & 0 & 0 & 51 & 51 \\
\hline Total & 4 & 6 & 1 & 51 & 62 \\
\hline
\end{tabular}

*N.A.= Não se aplica. Fonte: Autores. 
Verificou-se que 54 (87\%) puérperas alegaram que não enfrentam nenhuma dificuldade na amamentação. Ao considerar as puérperas que apresentaram dificuldade na amamentação, $6(9,7 \%)$ sentem dor ao amamentar e $2(3,3 \%)$ relataram algum tipo de fator emocional que influencia negativamente no ato de amamentar.

Vale ressaltar que todas as pesquisadas alegaram terem sido orientadas quanto aos benefícios do aleitamento materno e sobre os 10 passos para promover a amamentação, bem como 31 (50\%) afirmaram achar bom o ato de amamentar e 31 (50\%) relataram achar ótimo amamentar. Todos os recém-natos das puérperas do estudo permaneceram no alojamento conjunto durante todo o tempo de internação.

Foi realizada a distribuição das puérperas de acordo com o conhecimento sobre quando deve ser iniciada a mamada e quanto a sua duração adequada da mamada. Ao serem questionadas sobre qual a duração adequada da mamada, 55 (88,9\%) mulheres responderam que até o bebê querer, 3 puérperas $(5 \%)$ responderam que até tiver leite, 3 puérperas $(5 \%)$ não responderam e $1(1,1 \%)$ não sabiam qual a duração adequada da mamada (Figura 1).

Figura 1 - Distribuição de puérperas de acordo com o conhecimento de quando deve ser iniciada a amamentação e a duração adequada de uma mamada. Aracaju, SE, 2018.

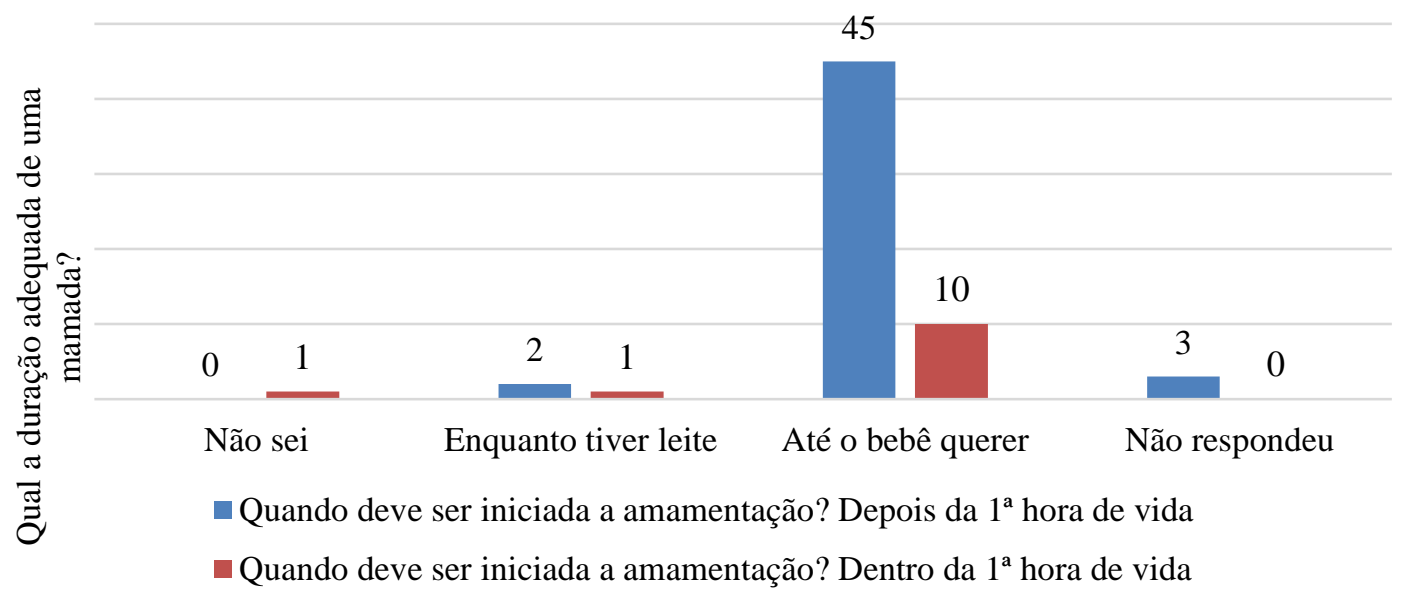

Fonte: Autores.

\section{Discussão}

A ação de pôr uma criança no mundo circunda não apenas a gestação mas sobretudo os cuidados perante o neoato e as modificações que demanda da mulher para se acomodar a essa atual conjuntura, tendo em vista que a lactação são acotecimentos inclusos nesse viver, com vinculos a essa etapa de acomodação. (Tomeleri et al. 2009).

A aleitação é um mecanismo que se amplia além da conexão materna se amplifica nas comunicações da e interrelações da mulher, sua percepção na lactação, as dificuldades, nas diversas esferas, sua emotividade. (Jimenez et al. 2017)

No entanto se faz necessário o entendimento das nutrizes em todas as suas esferas, compreendendo a sua feminilidade e a sua atuação como ser humamno. Os cuidados com o neonato, pode gerar ansiedade, especialmente no que diz respeito a amamentação, essas dificuldades carecem ser resolvidas pelo individuo que atuam no setor de asistência e a do seu recémnascido (Brasil, Ministério da Saúde. 2016)

A prática do AM é uma experiência que envolve fatores relacionados à criança e à mulher, a tomada de decisão em amamentar depende do que considera como prioritário para ela e seu filho (Silva, 2013), deste modo, a mulher nesse processo está continuamente avaliando os riscos e as vantagens de alimentar seu filho com o seu próprio leite. 
A dificuldade de compreensão dos reais motivos pelos quais muitas mulheres deixam de amamentar seus filhos é um dos grandes desafios do enfermeiro e da equipe multiprofissional para o alcance dos objetivos dos projetos e programas de incentivo ao AME. Desafio ainda maior configura-se a atuação junto a essas mulheres de intervir nos aspectos obscuros que levam à decisão materna de desmame e introdução de outros alimentos na dieta do recém-nascido (Gonçalves 2013).

O aleitamento não é uma prática fácil, exigindo uma adaptação da mulher ao seu novo papel de mulher-mãe. Fatores como falta de apoio, experiências negativas do aleitamento materno, retorno ao trabalho, problemas mamários, estado psíquico, falta de orientação, a falta de apoio da família dificulta o aleitamento materno.

As respostas sobre a importância do aleitamento materno foram pontuais, e sugerem nenhuma deficiência de um conhecimento abrangente sobre a importância do aleitamento materno, vantagens em relação a outros alimentos e adequação ao bebê em seus primeiros meses de vida, embora essas informações sejam frequentemente divulgadas por profissionais.

Sabe-se que o desmame precoce é resultado de uma complexa interação de fatores socioculturais, como por exemplo, o processo de industrialização, o surgimento e a divulgação de leites industrializados, com a respectiva adesão de profissionais de saúde à prescrição da alimentação artificial, a inserção da mulher no trabalho, e a adoção nas Maternidades de medidas pouco incentivadoras do aleitamento materno (World Health Organization, 1981).

O recém-nascido possui sistema imune ainda imaturo, sendo mais suscetível a infecções. O primeiro contato entre o bebê e anticorpos é a partir do aleitamento materno, a partir disto, se torna imprescindível a amamentação na proteção do bebê contra bactérias e vírus (Silva et al. 2020).

Ressalta-se a importância para o sucesso dos 10 passos do aleitamento materno, onde 100\% das puérperas entrevistadas confirmam que tem orientação de tais métodos. Essas orientações são de suma importância, pois é através dos dez passos que se garante um aleitamento materno exclusivo de qualidade.

Com vistas à redução do desmame precoce, e com base na modificação de rotinas hospitalares inadequadas à prática da amamentação, foram normatizadas pela Organização Mundial da Saúde (OMS) e Fundo das Nações Unidas para Infância UNICEF as orientações denominadas dos Dez Passos para o Sucesso do Aleitamento Materno.

O desmame pode desfardar modificações nas dimensões das mamas, peso e diversos sentimentos que podem ser benéficos ou desagradáveis devido o rompimento da lactação e mudanças hormonais (Brasil, Ministério da Saúde. 2017).

A deliberação em garantir a lactação até que a criança decida abandonar sem interferência cabe não apenas a mãe, mas a sua família, diversas são as causas implicada nessa resolução e é nesse momento que a atuação do profissional de saúde se faz necessária auxiliando nas tomadas de decisão após informar os prós e os contras.

Nesse contexto o profissional de enfermagem pode desempenar papel importante para amenizar problemas naturais envolvendo o aleitamento, divulgando os serviços para mulheres independentemente de serem gestantes, orientando sobre os benefícios do aleitamento materno exclusivo e incentivar a nutriz a amamentar seu filho.

Para Lobo et al. (2020), considera-se importante a qualificação e especialização dos profissionais de enfermagem quanto a amamentação, desta forma, os profissionais serão capazes de ofertar um melhor atendimento e acolhimento para as mães que se encontram no momento de lactação em âmbito hospitalar.

\section{Conclusão}

Evidenciou-se que a maternidade e a equipe multiprofissional que nela atuam, estão inseridos no processo para prevenir possíveis causas do desmame precoce, como o ingurgitamento mamário e as fissuras. Assim, como existe o apoio emocional e profissional às puérperas, a abrangência da prática do aleitamento materno na amostra do estudo mostrou-se adequada, demonstrando o excelente trabalho desenvolvido no cenário do estudo. 
A orientação adequada das mães sobre o AM é importante para o sucesso da amamentação no período pós-parto, amenizando possíveis problemas como dor na amamentação e desmame precoce.

Desta forma, torna-se indispensável a realização de trabalhos que abordem condutas de profissionais da saúde quanto ao aleitamento materno,

\section{Referências}

Almeida, I. S., et al. (2010). Amamentação para mães primíparas: perspectivas e intencionalidades do enfermeiro ao orientar. Cogitare Enfermagem. 15(1), $19-25$.

Brasil. Ministério da Saúde. (2012). Aleitamento materno, distribuição de fórmulas infantis em estabelecimentos de saúde e a legislação. Caderno de Atenção Básica $n^{o} 1$ / Departamento de Ações Programáticas e Estratégicas.

Brasil. Ministério da saúde. (2016). Protocolos da Atenção Básica: saúde das mulheres. Brasília, DF.

Brasil. Ministério da Saúde. (2017). Bases para a discussão da Política Nacional de Promoção, Proteção e Apoio ao Aleitamento Materno. Secretaria de Atenção à Saúde. Departamento de Ações Programáticas Estratégicas.

Del Castanhel, M. S. et al. (2016). Promoção do leite materno na atenção básica. Universidade Federal de Santa Catarina (UFSC) - Formação para Atenção Básica.

Fundo das Nações Unidas para Infância (UNICEF) (2008). Iniciativa Hospital Amigo da Criança. Módulo I histórico e implementação.

Gonçalves P. (2013). Assistência de enfermagem ao incentivo aleitamento materno frente as dificuldades apresentadas por primíparas no alojamento conjunto. Universidade do Estado do Mato Grosso (UNEMT). 1, 13-14.

Jimenez, B. C. et al. (2017). Beneficios a corto, medio y largo plazo de la ingesta de leche humana en recién nacidos de muy bajo peso. Nutr. Hosp. 34(5).

Lobo, B. M. I. D. S. (2020). Estratégias de acolhimento à nutriz utilizadas pelos profissionais de saúde: uma revisão integrativa. Research, Society and Development. $9(8)$, e13985168.

Mendes, K. D. D., Silveira, R. C. C. P. \& Galvão, C. M. (2008). Revisão integrativa: método de pesquisa para a incorporação de evidências na saúde e na enfermagem. Texto \& contexto Enfermagem, 17, 758-764.

Miot, H. A. (2011). Tamanho da amostra em estudos clínicos e experimentais. Jornal Vascular Brasileiro. 10 (4), $275-8$.

Moura, M. A. V., Costa, G. R. M., \&Teixeira, S. C. (2010). Momentos de verdade da assistência de enfermagem a puérpera: um enfoque na qualidade. Rev. enfermagem UERJ. (1), 429-434.

Narchi, N. Z. et al. (2009). Variáveis que influenciam a manutenção do aleitamento materno exclusivo. Revista da Escola de Enfermagem da USP. 43(1), 8794.

Organização Mundial da Saúde (OMS). (2001). Evidências Científicas dos Dez Passos para o Sucesso do Aleitamento materno.

Secretaria Municipal da Saúde Departamento de Atenção à Saúde das Pessoas Programa de Aleitamento Materno. (2020). Protocolo E Diretrizes De Atendimento Em Aleitamento Materno, Prefeitura Municipal de Ribeirão Preto.

Silva IA. (2013). O significado de amamentar para a mulher. In: Merighi MPB, Abordagens teórico-metodológicas qualitativas: a vivência da mulher no período reprodutivo. Rio de Janeiro, Guanabara Koogan. (12), 135-51.

Silva, D.I.S., et al. (2020). A importância do aleitamento materno na imunidade do recém-nascido. Research, Society and Development. 9(7), e664974629.

Starpasson, R.S.L. (2010). Repercussões Materno Fetais e Neonatais da doença de graves na gestação; Rev. Universidade Federal do Paraná. (1), 101-102.

Tomeleri, K. R. \& Marcon, S. S. (2009). Mãe adolescente cuidando do filho na primeira semana de vida. Rev. Bras. Enfermagem. 62(3), 355-361.

Victora, C. G. et al. (2016). Breastfeeding in the 21st century: epidemiology, mechanisms, and lifelong effect. The Lancet. 387(10017), 475-490.

WHO (World Health Organization). (1981). The international code of marketing of breast-milk substitutes. Genebra: The Organization. 